\title{
Microscopic Characterization of Dendrimer-Derived Bimetallic Pt-Cu Catalysts
}

\author{
H. Xie, D. Liu, C.T. Williams, H. J. Ploehn, and J. Y. Howe* \\ Department of Chemical Engineering, University of South Carolina, Columbia, SC 29208 \\ *Metal and Ceramics Division, Oak Ridge National Laboratory, Oak Ridge, TN 38831
}

Bimetallic catalysts have found very important applications in large-scale industrial processes such as naphtha reforming, automobile exhaust clean-up, and direct methanol fuel cells [1]. The catalytic behavior of bimetallic catalysts is determined by its structure, which varies significantly with the preparation method. Conventional preparation methods utilizing inorganic salt precursor have met limited success in control the size and composition. A new approach is to use dendrimer-metal nanocomposites as a precursor to formation of nanoparticles on high surface area supports. Dendrimers are mono-dispersed, hyper-branched polymers that emanate from a central core with repetitive branching unit [2]. While possessing a very dense exterior, they contain hollow pockets as ideal nanoscale containers. They can exert control over size and compositions of nanoparticles, which in principle can allow for tuning of catalytic properties.

In this work, we have synthesized colloid particles of platinum $\left(\mathrm{Pt}_{20}\right)$, copper $\left(\mathrm{Cu}_{20}\right)$ and bimetallic platinum-copper with $\mathrm{Pt} / \mathrm{Cu}$ atomic ratio of $1\left(\mathrm{Pt}_{20} \mathrm{Cu}_{20}\right)$ and $2\left(\mathrm{Pt}_{20} \mathrm{Cu}_{10}\right)$ in PAMAM $\mathrm{G} 4 \mathrm{OH}$ dendrimer aqueous solution by reducing corresponding dendimer-metal nanocomposites with hydrogen via a co-complex and co-reduction synthesis method [3]. We further made catalysts supported on silica with these nanoparticles and explored an optimized protocol for dendrimer decomposition, metal exposure, and activation for these catalysts using transmission FTIR specteoscopy. Corresponding conventional catalysts were synthesized and characterized for comparison.

We report TEM and energy dispersive spectroscopy (EDS) studies on dendrimer-derived and conventional bimetallic Pt-Cu catalysts treated with our optimized protocol. TEM measurements were carried out using both Hitachi HF2000 FE TEM and HD2000 STEM, with specimens supported on holey carbon film-coated nickle grids. It is our interest to find out the particle size and distribution, along with the compositional variation in these catalysts.

Histograms of particle size distributions were obtained through measurement of at least 200 randomly selected particles from at least 5 images of any given sample. Representative TEM images and particle size distributions of $\mathrm{Pt}_{20} \mathrm{Cu}_{20}$ bimetallic nanoparticles are shown in Figure 1 . The particles are nearly spherical. Analysis reveals that Pt-Cu bimetallic nanoparticles have an average diameter of about $2.2 \mathrm{~nm}$ for the dendrimer-derived catalyst, while $2.9 \mathrm{~nm}$ for the conventional catalyst. In addition, EDS of these dendrimer-derived nanoparticles suggests that they are bimetallic in nature. In both catalysts, there are significantly larger particles ( $>4 \mathrm{~nm}$ ) resulting from the catalyst treatment. However, there are many more large particles in the conventionally prepared catalyst, as indicated in the histogram showing a log-normal distribution. Through the EDS analysis, we also found that the relative ratio of copper to platinum varies as a function of particle size. In some specimens, smaller sized particles have a higher concentration of platinum than copper. Whereas the larger particles contain more copper. Coupled with similar microscopic information obtained for related catalysts (e.g., $\mathrm{Pt}_{20} \mathrm{Cu}_{10}, \mathrm{Pt}_{20}$ ), this data suggests that the $\mathrm{G} 4 \mathrm{OH}$ dendrimer is capable of exerting significant control over particle formation and sintering on the catalyst support [4].

[1] Rodriguez, J.A.; Goodman, D.W. J. Phys. Chem. (1991),95(11), 4196-206

[2] Tomalia, D.; Naylor, A.; Doddard, W. Angew. Chem . Int. Ed. Engl. (1990), 29, 138-175. 
[3] To clarify notation, e.g., $\mathrm{Pt}_{20}$ means the molar ratio G4-OH PAMAM:Pt $=1: 20$

[4] Research at the Oak Ridge National Laboratory (ORNL) High Temperature Materials Laboratory (HTML) was sponsored by the Assistant Secretary for Energy-Efficiency and Renewable Energy, Office of FreedomCAR and Vehicle Technologies, U.S. Department of Energy under contract DE-AC05-00OR22725 with UT-Battelle, LLC.

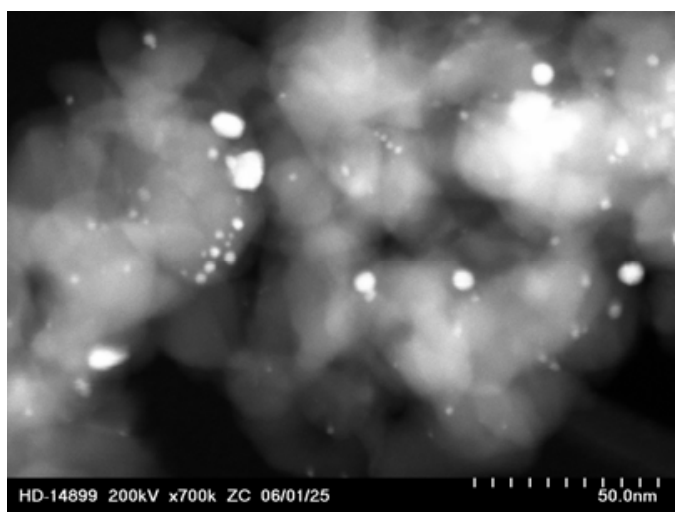

(a)

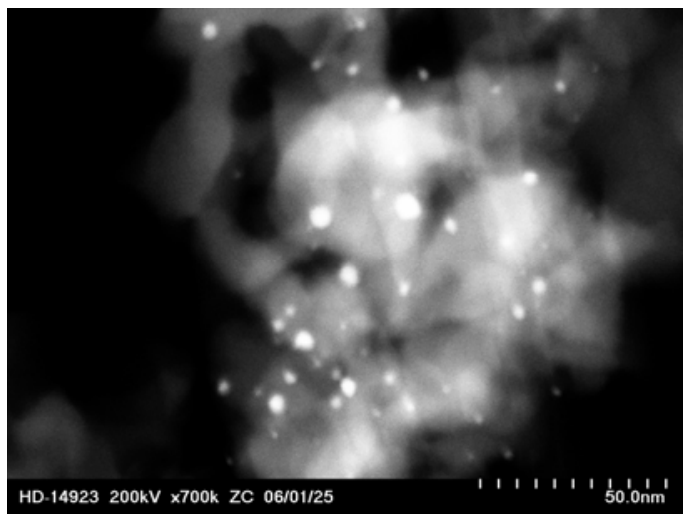

(b)
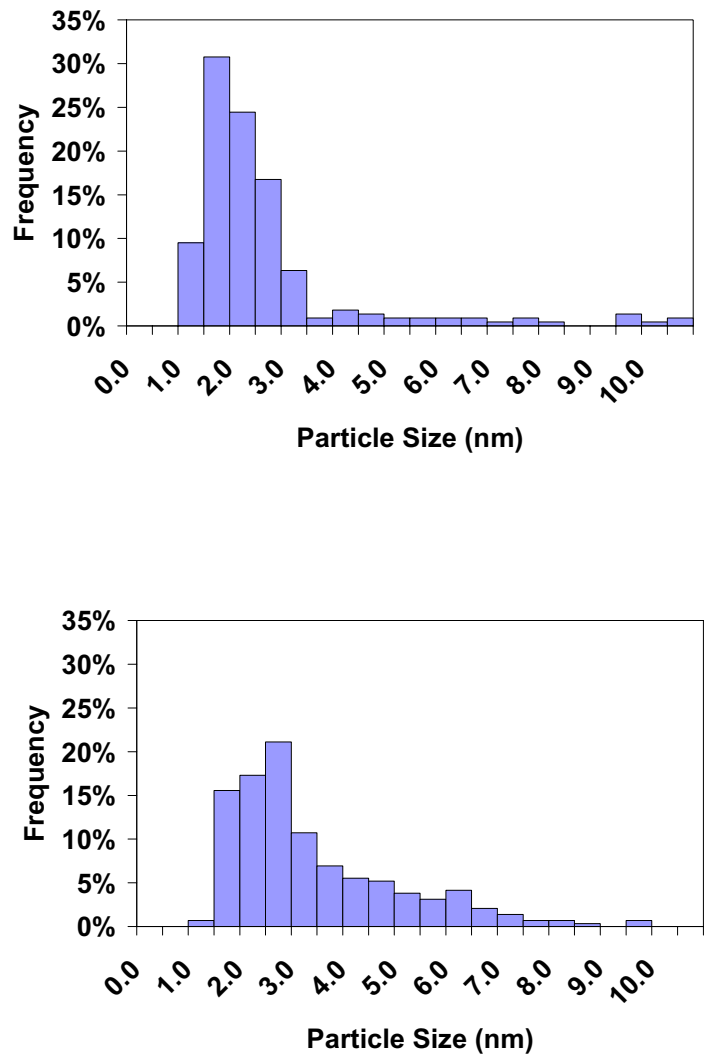

Figure 1. particle size distribution histogram for (a) $\mathrm{Pt}_{20} \mathrm{Cu}_{20}$ dendrimer-derived catalyst based on 221 randomly selected particles and (b) $\mathrm{Pt}_{20} \mathrm{Cu}_{20}$ conventional catalyst based on 289 randomly selected particles 